\title{
Research on the Effects of Spatial Cognition by Emulation Model and Perspective Model in MCT
}

\author{
Li Ping \\ Mechanical and Power Engineering College, Harbin University of Science and \\ Technology, Harbin 150080, China \\ 1198719546@qq.com
}

\begin{abstract}
Affecting the effects of MCT evaluation of spatial cognition has a lot of factors. In problems of shape discriminate, when using a simulation model of MCT instead of perspective MCT model accuracy improved. In problems of quantity discriminate, by comparing the simulation model of MCT and perspective model of MCT, correct answer no bigger difference. Produced with the help of computer simulation model can help students to comprehend, but three-dimensional space cognitive enhancement is based on the experience of students as the main body understanding and the result of rational thinking.
\end{abstract}

Keywords: engineering drawing, emulation model, perspective model, the effect of spatial cognition

\section{Introduction}

The students' space cognition ability is associated with graphics education effects. MCT (Mental Cutting Test: MCT) because the real test of cutting surface can quantitatively evaluate spatial cognition and graphics education, it is widely used [1]. Figure 1 shows the MCT, which uses perspective view to signify the solid and the cutting surface. We choose the answer to the question from five cutting graphics. The number of questions is 25 and answer time is 20 minutes.

The 25 questions of MCT can be divided into problems of shape discriminate and quantity discriminate. Among them, the amount of the "quantity" judgment is $32 \%$ and the "shape" judgment is $68 \%$. The "shape" judgment is how to judge the shape of cutting surface correctly. The "quantity" judgment is to choose the right answer from similar graphics after cutting, which not only judge the shape of section but also judge the length and angle of the ridge.

In the process of testing and evaluating spatial cognitive ability according to the MCT, there are many factors can affect the results such as eyes visual error, the proportion of test types, the characteristics of the three-dimensional model, etc., [2, 3]. This paper focuses on study the effect of different kinds of the characteristic three-dimensional model on the accuracy of MCT tests, studying subjects' solution process and reasons of wrong answer, which well reveals the MCT cutting real shape test for quantitatively evaluate the effect of spatial cognition. This paper analyzed evaluation mechanism of spatial cognition ability by MCT.

\section{Research Methods}

(1) The testers are six classes' students from the mechanical engineering of university of science and engineering, they are divided into two groups and do the tests within one week after go to college. 
(2) Research methods are written tests. A kind of MCT test is use perspective type to express the three-dimension and cutting surface. Another kind of MCT test is to use 3D software to draw the simulation model of cut cube which is similar to perspective MCT to replace the stereogram of examination.

As shown in Figure 2. Distribute test paper to two groups of students and answer it at the same time, as shown in Figure 2.

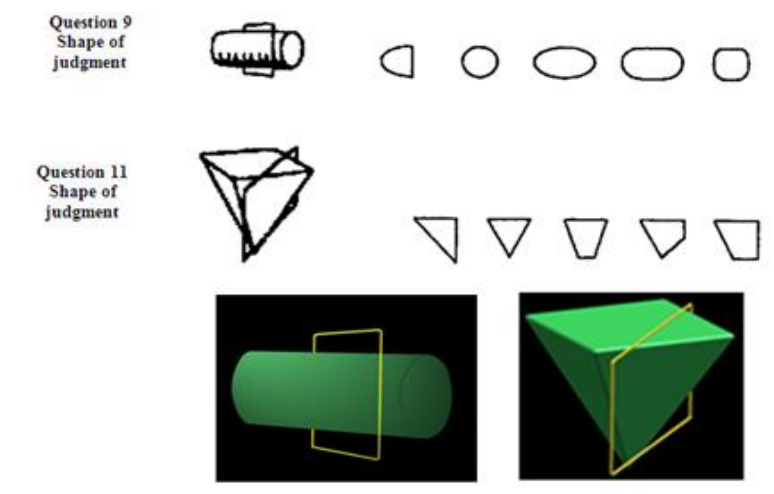

Figure 1. The Perspective Diagram and Simulation Diagram of Shape Judgment

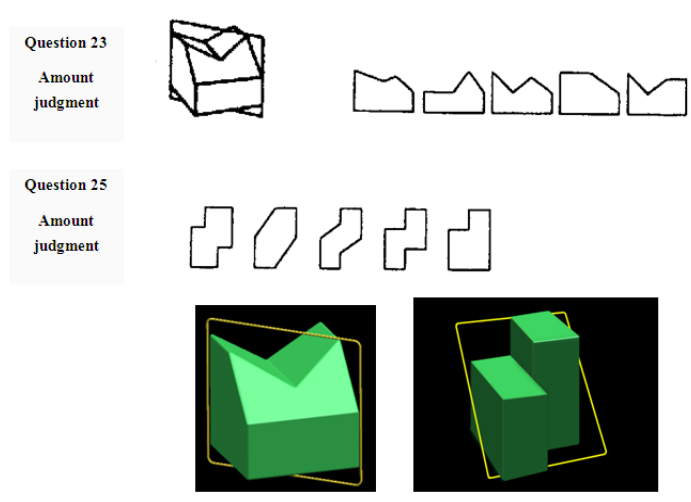

Figure 2. The Perspective Diagram and Simulation Diagram of Quantity Judgment

\section{Results and Analysis}

Using the SPSS statistical software to analyze and discuss two kinds of test data.

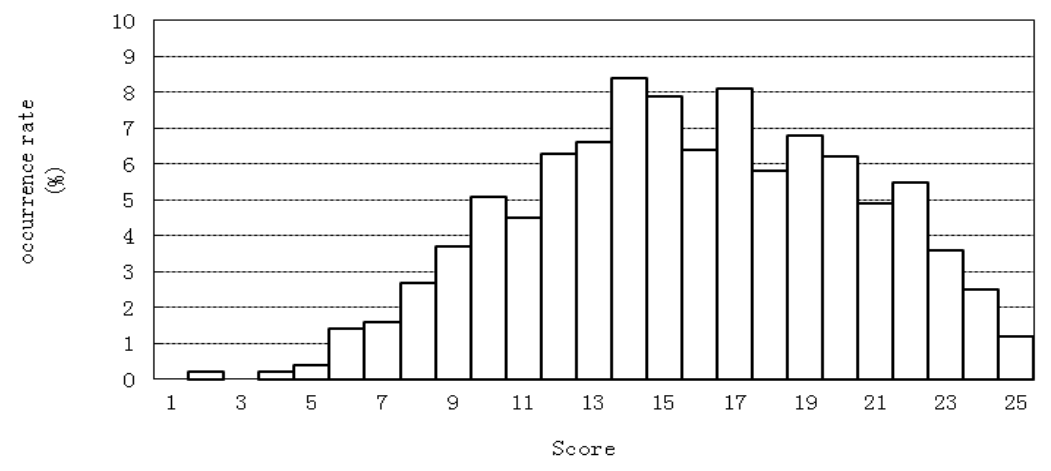

Figure 3. Perspective Distributions of the Score 


\subsection{The Distribution of Perspective Score}

Figure 3 is the distribution of perspective MCT score. As shown in the figure, the scope of score ranges from 1 to 25 . Deviation degree is-0.10, kurtosis is 2.32 , the average score is 15.8 , the standard deviation is 4.6.

(Score levels are all sorts of MCT using percentage to segment score. Low score layer is 0 to 11 points, middle score layer is 12 to 20 points, high score layer is $21-25$ points.)

The average score is 15.8 points, the standard deviation is 4.6 points. The average score 15.8 points plus the standard deviation 4.6 points equal to 21 points. The above is called high score. The average score 15.8 points minus the standard deviation 4.6 points equal to 11 points. The below is called low score.

\subsection{Accuracy of All the Problems}

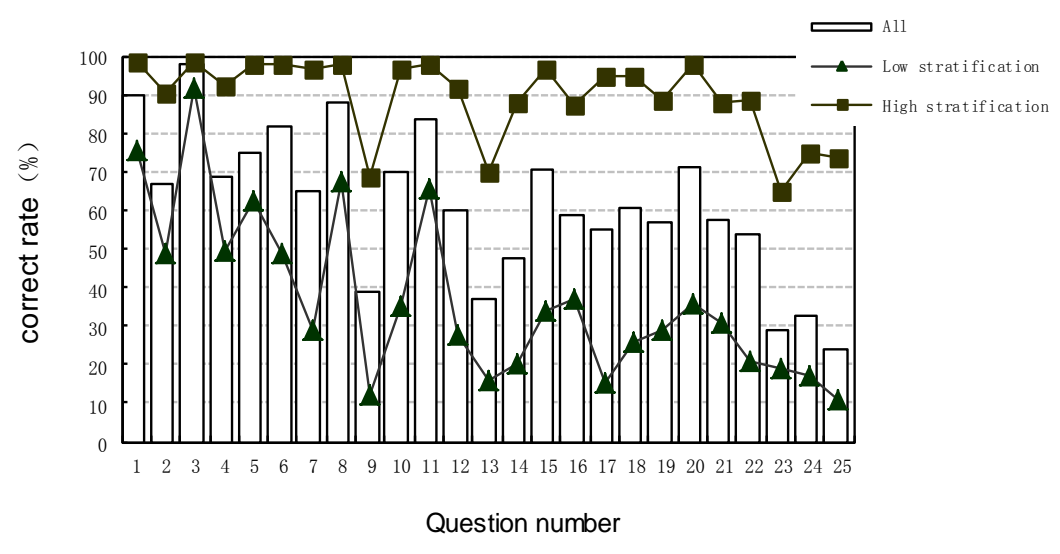

Figure 4. Accuracy of Each Question in Perspective Models' Average, High and Low Scores

Accuracy is the percentage of the number of all the correct answer divided by the number of all testers. Figure 4 shows the number of question arrange in order in horizontal axis, Y-axis is accuracy. The average accuracy of each question ranges from $24 \%$ to $99 \%$ in amplitude distribution. Figure 4 also shows the difference of difficulty of each question. The curve in figure 4 also expresses the accuracy of higher layer and lower layer. Whichever experimental group orders are the same. These problems' relative difficulty also shows the same order even in high scores and low scores.

Comparing high layers, accuracy of question 23, 9, 13 are low, which are difficult problems. Accuracy of question 3, 1, 8, 5, 11 are high. In low layers, it can be considered as low difficult problems.

\subsection{Analysis of High and Low Difficult Problems}

Low and middle difficult problems are shape discriminate problem $3,1,8,5$ in test problems, most of them are a variety of cubes lack of a corner and a quarter of the cylinder. The cutting cube problem is relatively simple three-dimensional cutting problem, which is easy to understand.

Problem 25, 9, 24 are shape discriminate problems in high difficult problems, question 23,13 are typical quantity judge problems; In general, judge the length of the quantity in the picture is difficult.

We can see the accuracy in figure 4, tend of high scores and low scores have a very big difference. In the shape judge problems, shape is correct in high scores and wrong in low scores, which is the difference in solution process. In quantity judge problems, different quantity selections have more errors in high scores. Not only different quantity selections 
but also shape selections in low scores have more error than that in high scores. This result also shows that there are quantity judge problems in low scores and much error in shape discriminate stage.

\section{Comparatively Analysis Scores of the Simulation Model and the Perspective Model}

When using simulated three-dimensional stereo replace perspective three-dimensional stereo in the same paper, the two kinds of MCT test scores' accuracy are shown in Figure 5 .

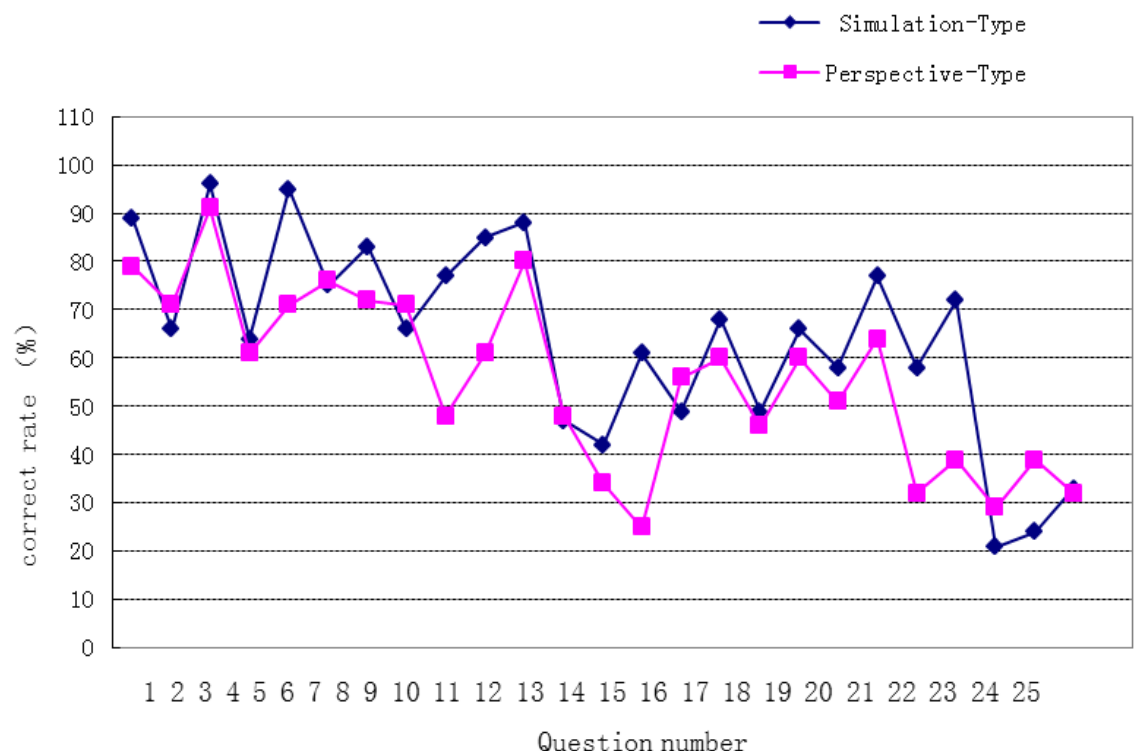

Figure 5. The Correctness of MCT in Simulation-Type and Perspective-

The average score of Simulated MCT is 17.07 points (SD3.09) and the average score of perspective MCT is 15.8 points (SD3.88), there are differences. From here, we can see that using the simulated three-dimensional stereo to represent three-dimensional stereo in MCT, MCT scores tend to rise. For each question' accuracy, question 9, 11 simulated MCT have higher score than perspective MCT. On the contrary, question 23, 25 become low. Question 9, 11 are shape discriminative problem, problem 23 is quantity discriminative problem. The climbing rate of high difficult problem and low difficult problem doesn't have particularly evident rule. So we can think: from overall comparing the three-dimensional view of MCT, shape discriminative problem' accuracy goes up larger, quantity discriminative problem has no big changes.

In the shape discriminative problems, high score sections can correctly solve different problems which are different from the perspective MCT. Even the low scorers have simulated model, the accuracy is improved.

Maybe due to simulated object model vividly suggest the position of cutting plane, it is easy to recognize and judge relative position of the three-dimensional and cutting plane and the shape of the three-dimensional (concave and convex surface).

Graphical solid of the perspective MCT, because its line is concise, comparing with the two-dimensional plan, it doesn't not like simulated solid, which can work out section from the solid consist of shadows and texture surface. It need to abstract the solid structure and then determine ridge and solve the problem. From many shape judging problems of MCT, we can learn the main problems are generate three-dimensional graphics from (perspective) figures. 
Judging from quantity judgment, it does not reflect large differences of answer accuracy between the simulated MCT and perspective MCT. It does not have link with the improvement of the more difficult quantity judgment error. It is probably that the quantity judge problem is not as intuitive as shape judgment; it needs to use analytical thought to reveal the answer. In order to answer MCT correctly, when watching the problem diagram, we should not only capture the three-dimensional of the solid but also consider the solid processing such as height, length of the ridge.

In quantity judge problems we can't see the solution differences between the twodimensional MCT and the simulated MCT; this is because the solutions of quantity judgment actually reveal the investigation of analysis the quantity is necessary premise. This paper argues the above results illustrate that using teaching model display and animation demonstration of multimedia courseware in graphics teaching, which can let students get perceptual knowledge. But only using simulated model reminds the problem, it will not easy for the students fully understand. It is always that the simulated object model only applied as a means to help students understand, we students should understanding solid attentively in conscious, and only in this way can we reach a rational leap in the process of thinking.

\section{Conclusions}

The development of simulation of the three-dimensional view MCT, perspective MCT is prepared condition. According to the comparative study we can make following conclusions:

1. The average score of simulated three-dimensional MCT has improved. Simulated MCT and perspective MCT problems tend to choose the answer, it is easier to judge relative location of solid and cut surface of simulated three-dimensional mode, the judgment of the basic bump shape of solid is easier and the average score also rise.

2 . In the typical quantity judgment, reducing examples of quantity misjudged judgment couldn't improve accuracy. This is because we must analysis and study the characteristics when answer quantity judged problem. It also suggests that rational analysis thinking can reveal the answer in quantity judged problem.

In the simulated model of MCT we can perceive the deep-seated aspects of solid, when preparing the shadow, eyes parallax, texture elements, recognition about eyes parallax three-dimensional shape and relative position of solid and cutting section become easier. So we can see the accuracy of shape judgment questions rising in the simulated model of MCT, which means the shape judgment problems mainly use intuition to solve.

In a word, compare the two kinds of solid model tests and analysis solution process and reasons of wrong answer. The results: MCT mainly reflects operate ability to transform two-dimensional figure into three-dimensional stereoscopic image. It also reflects the processing, analysis, and inspection ability. This ability can be cultivated with the aid of computer simulated model to help students understanding two-dimensional graphics, but the improvement of three-dimensional space ability is based on the experience of understanding of the students as main body and the results of rational thinking.

\section{Acknowledgements}

This research was supported by the Heilongjiang Province Heilongjiang Province Education Science "twelfth five-year" Plan Project (Grant No. GBC1211058).

\section{Reference}

[1] T. Saito, K. Makino, K. Shiina and T. Jingu, "Causes of Error in a Mental Cutting Test-Analysis of Problem Solving Process by the Use of Eye Fixations Data(2)", Proc. 6h ICECGDG, Tokyo (Japan), (1999), pp. 815-819.

[2] G. Adancz and A. Valsco, "Predicting Academic Success of Engineering Students in Technical Drawing from Visualization Test Scores", journal of Geobimetry and Graphics, vol. 6, no. 1, (2012), pp. 99-109. 
[3] K. Suzukl, S. Waktta and S. Nagano, "Improvement of Spatial Ability through Graphics Education, Proc.”, 4th ICEGDG Miami, (2010), pp. 442-448.

[4] K. Nishihara, K. S. Nishihara, I. Sakamoto, K. Chibana, K. Yoshida, "Effect. Of Hidden Line Removal on the Score of Mental Cutting Test Remodeled by Anaglyphic Representation", Proceedings of 6th. ICECGDG, Tokyo, (2008), pp.786-790.

[5] K. Suzuki, "Activities of the Japan Society for Graphic Science Research and Education", 10th ICGG, vol. 3, (2009), pp. 1-3.

[6] E. Tsutsumi, H. Stachel, G. Weiss, "Hans-Peter SCHROCKER, Evaluation of Students' Spatial Abilities in Austria and Germany, 11th ICGG, (2007), pp. 198-202.

[7] T. Saito, K. Makino, K. Suiina, K. Suzuki and T. Jingu, "Cause of Error a Mental Cutting Test- Analysis of Problem Solving Process by the Use of Eye Fixation Data(2), Proceedings of the 6th International Conference on Engineering Compute Graphics and Descriptive Geometry, (2006), pp, 560-573.

[8] Y. D. Zhang, J. X. Jiang, "Bending Property Analysis and Experimental Study of Orthodontic Wires", Chinese Mechanical Engineering, vol. 22, no. 15, (2011), pp. 1827-1831.

[9] J. Jingang, H. Tianhua, D. Hongwei and L. Bin, "Electric field structure analysis and experimentation of needle-plate type electro spinning machine", International Journal of Control and Automation, vol. 7, no. 1, (2014), pp. 369-378.

[10] D. Hongwei and J. Jingang, "Experimentation and finite element analysis of electric field structure of electro spinning machine", Advances in Intelligent and Soft Computing, vol. 105, (2011), pp. 283-289. 